\title{
A comment on effect of fluoride exposure on the intelligence of school children in Madhya Pradesh, India
}

Sir,

This is in reference to the article titled, "Effect of fluoride exposure on the intelligence of school children in Madhya Pradesh, India" published in Volume: 3 (2) of your journal. ${ }^{[1]}$ The authors have done a decent enough work to draw attention toward fluoride exposure and level of intelligence among students. Here, I would like to draw the attention of authors to following points: (1) Table 1 shows that mean years of education of heads of family is the lowest in families wherein the water fluoride level is the highest $(6.71 \pm 3.12)$ which in itself needs a discussion in the publication. As rightly pointed point out by the authors in discussion part, studies have shown the education level of parents, SES of the family, nutrition, and iodine deficiency affect the intellectual ability of children. The association between water fluoride levels and intellectual abilities may be confounded by low educational levels and hence socio-economic status. Did the authors perform any analyses to adjust for confounding? (2) Again Table 1 shows that height for weight for children is the lowest in families wherein the water fluoride level is the highest $(2.24 \pm 0.85)$. As we are aware that height for weight is a good indicator for presence of chronic malnutrition, a factor that may again influence the intellect of children, this also needs some deliberation. Last, I would like to know as to whether there is a cutoff of the water fluoride levels at which intellectual deterioration starts or not.

Table 1: Details of fluoride levels

\begin{tabular}{|c|c|c|c|c|c|c|}
\hline \multicolumn{7}{|c|}{ Water fluoride level (PPM) } \\
\hline & $<1.5(n=50)$ & $<1.5-3.0(\mathrm{n}=39)$ & $3.1-4.5(n=43)$ & $>4.5(n=38)$ & $\begin{array}{l}\text { ANOVA } \\
\text { (F) value }\end{array}$ & $P$ value \\
\hline $\begin{array}{l}\text { Urinary fluoride (PPM. } \\
\text { Meam } \pm \mathrm{SD} / \text { range) }\end{array}$ & $\begin{array}{l}2.25 \pm 0.28 \\
(1.7-2.8) \neq\end{array}$ & $\begin{array}{l}3.28 \pm 0.48 \\
(2.5-4.3) \neq\end{array}$ & $\begin{array}{l}4.85 \pm 0.50 \\
(4.1-6.0) \neq\end{array}$ & $\begin{array}{l}7.01 \pm 1.02 \\
(5.6-8.4) \neq\end{array}$ & 486.339 & 0.000 \\
\hline $\begin{array}{l}\text { Urinary iodline }(\mu \mathrm{g} / \mathrm{l} \text {, } \\
\text { Mean } \pm \mathrm{SD} / \mathrm{range})\end{array}$ & $\begin{array}{l}2.75 .83 \pm 63.34 \\
(231.2-396.7)\end{array}$ & $\begin{array}{l}275.04 \pm 62.80 \\
(228.8-392.4) \neq\end{array}$ & $\begin{array}{l}275.51 \pm 63.48 \\
(237.5-400.2) \neq\end{array}$ & $\begin{array}{l}277.97 \pm 65.17 \\
(234.0-402.3) \neq\end{array}$ & 0.016 & 0.997 \\
\hline $\begin{array}{l}\text { Urinary lead }(\mu \mathrm{g} / \mathrm{l}, \\
\text { Mean } \pm \mathrm{SD} / \mathrm{range})\end{array}$ & $\begin{array}{l}32.12 \pm 3.40 \\
(26.6-37.0) \neq\end{array}$ & $\begin{array}{l}31.69 \pm 3.58 \\
(25.8-37.5) \neq\end{array}$ & $\begin{array}{l}31.88 \pm 3.57 \\
(26.3-35.4) \neq\end{array}$ & $\begin{array}{l}32.29 \pm 3.35 \\
(27.9-34.7) \neq\end{array}$ & 0.229 & 0.876 \\
\hline $\begin{array}{l}\text { Urinary arsenic }(\mu \mathrm{g} / \mathrm{l} \text {, } \\
\text { Mean } \pm \mathrm{SD} / \text { range })\end{array}$ & $\begin{array}{l}5.74 \pm 2.95 \\
(0.0-8.8) \neq\end{array}$ & $\begin{array}{l}6.13 \pm 2.83 \\
(0.0-8.2) \neq\end{array}$ & $\begin{array}{l}6.21 \pm 2.97 \\
(0.0-9.0) \neq\end{array}$ & $\begin{array}{l}6.34 \pm 2.42 \\
(0.0-7.9)_{\mp}\end{array}$ & 0.385 & 0.764 \\
\hline
\end{tabular}

\# Range 


\section{Sunil Kumar Raina}

Department of Community Medicine, DR. RPGMC, Tanda, Kangra (Himachal Pradesh), India

Address for correspondence: Dr. Sunil Kumar Raina, Department of Community Medicine, DR. RPGMC, Tanda, Kangra (Himachal Pradesh), India. E-mail: ojasrainasunil@yahoo.co.in

\section{Reference}

1. Saxena S, Sahay A, Goel P. Effect of fluoride exposure on the intelligence of school children in Madhya Pradesh, India. J Neurosci Rural Pract 2012;3:144-9.

\begin{tabular}{|l|l|}
\hline \multicolumn{2}{|c|}{ Access this article online } \\
\hline Quick Response Code: \\
\hline
\end{tabular}

